\title{
Instrumentation Time Efficiency of Rotary and Hand Instrumentation Performed on Vital and Necrotic Human Primary Teeth: A Randomized Clinical Trial
}

\section{Vieyra JP* and Enriquez FJJ}

School of Dentistry, Universidad Autónoma de Baja California, Campus Tijuana, Tijuana, Baja California, México

\begin{abstract}
Aim: To compare the instrumentation time efficiency of rotary and hand instrumentation performed on necrotic human primary teeth.

Methodology: Patients aged 4-7 years were enrolled, forty five teeth (19 maxillary and 26 mandibular teeth), which had a total of 102 canals and completely formed apices and of minimum $10 \mathrm{~mm}$ root length were selected. Of the 45 treated primary molars, 31 teeth were diagnosed as having chronic pulpitis, and 14 as having pulp necrosis that responded negative to hot and cold tests; and, clinically, all pulps were confirmed to be necrotic on entrance into the pulp chamber.

Results: The mean time spent for rotary root canal preparation and hand preparation for the three groups was GI: $20.10 \pm 7.86$, GII: $9.37 \pm 2.19$ minutes and GIII: $10.45 \pm 4.77$ minutes, respectively. With regard to canal filling quality, 29 cases $(64.44 \%)$ were flush-filled, 5 cases $(11.11 \%)$ were under-filled, and 11 cases $(24.44 \%)$ were over-filled. The Student $t$ test was used to compare data whether there were statistically significant differences between the results obtained clinically. Significance was set at $p<0.05$.

Conclusion: Clinically, time efficacy in primary molar endodontic treatment, especially with the unpredictability and difficulty of canal morphology, is invaluable. The use of rotary files in primary teeth has several advantages when compared with manual $\mathrm{K}$ files.
\end{abstract}

Keywords: Root canal treatment; Instrumentation; Primary tooth; Root canal preparation; EAL; Endo Vac

\section{Introduction}

The goal of pulp therapy in the primary dentition is to retain the primary tooth as a fully functional part of the dentition, allowing at the same time for mastication, phonation, swallowing, and the preservation of the space required for the eruption of the permanent tooth $[1,2]$. The premature loss of permanent teeth. Maintenance of primary teeth until physiological of primary teeth may cause changes in the chronology and sequence of eruption exfoliation prevents deleterious habits in children [3].

The primary objectives of cleaning and shaping the root canal system are removing soft and hard tissue containing bacteria, providing a path for irrigants to the apical third, supplying space for medicaments and subsequent obturation, retaining the integrity of radicular structure [1].

The choice between pulpotomy and pulpectomy is generally based on the severity of the symptoms clinically and/or radiographically. When indicated, the primary tooth pulpotomy is a relatively simple procedure with generally good clinical results [3].

Moreover, pulpectomy are treatment stressful for the child and more complicated due to the anatomical complexity which is not in permanent tooth. Pulpectomy is a heavier treatment for the child and is more complicated due to anatomical complexities that are not found in the permanent tooth [3].

In addition to these, in primary teeth it is important to preserve the tooth until its natural exfoliation time, thus preserving arch integrity. The premature loss of primary teeth may cause changes in the chronology and sequence of eruption of permanent teeth. Maintenance of primary teeth until physiological exfoliation contributes to mastication, phonation and aesthetics and prevents deleterious habits in children [2].
Vital pulp therapy includes 2 therapeutic approaches: indirect pulp treatment (IPT) in cases of deep dentinal cavities and direct pulp capping (DPC) or pulpotomy in cases of pulp exposure [1].

According to the Guidelines of the American Academy of Pediatric Dentistry pulpectomy is indicated in primary teeth with carious pulp exposures in which, following coronal pulp amputation, the radicular pulp exhibits clinical signs of hyperaemia, or evidence of necrosis of the radicular pulp with or without caries involvement [4].

Success of pulpectomy depends on elimination of irritants by means of cleaning and shaping the root canal and it is dependent on microbial reduction as a result of chemomechanical preparation [4].

Furthermore, maintaining esthetics will avoid psychological problems related to the loss of teeth. Clinically, the choice of pulp therapy is based on semiology despite the assessment difficulties and imprecision of pulp tests related to deciduous dentition [4]. Reluctance to carry out root canal treatment (RCT) is based on the difficulty to clean and shape the curved root canals of the primary molars [3].

Pulpectomy on primary teeth with severe pulpal involvement should be considered as a treatment of choice [5]. Clinical success occurs when the tooth is painless, firm, non-mobility, and without any signs

*Corresponding author: Jorge Paredes Vieyra, PMB\#1513, 710E San Ysidro Blvd. suite "A", San Ysidro California 92173, USA, Tel: (619) 8463512, 01152664 1230750; E-mail: jorgitoparedesvieyra@hotmail.com

Received March 04, 2014; Accepted March 29, 2014; Published March 31, 2014

Citation: Vieyra JP, Enriquez FJJ (2014) Instrumentation Time Efficiency of Rotary and Hand Instrumentation Performed on Vital and Necrotic Human Primary Teeth A Randomized Clinical Trial. Dentistry 4: 214. doi:10.4172/2161-1122.1000214

Copyright: $\odot 2014$ Vieyra JP, et al. This is an open-access article distributed under the terms of the Creative Commons Attribution License, which permits unrestricted use, distribution, and reproduction in any medium, provided the original author and source are credited. 
of inflammation or infection [6]. The conventional instrumentation technique for primary teeth remains hand instrumentation which is time-consuming [7].

Root canal filling in primary molars is contraindicated in teeth with: (1) non-restorable crowns (2) perforation to the pulpal floor (3) reduced bone support and/or extreme tooth mobility (4) radiographic indication of extensive internal or external root resorption (5) periradicular radiolucency involving the follicle of the permanent tooth (6) underlying dentigerous or follicular cysts, and (7) in medically compromised children [6,7]

A practical pulpectomy technique for the primary dentition should include the following features: a) fast and simple procedures, with short treatment times and a minimal number of appointments; b) effective debridement of the root canals without weakening the tooth structure or endangering the underlying permanent teeth; c) few procedural complications; and d) maintaining tooth function until it is naturally shed [8].

The majority of studies reported significant reduction of bacteria with an increase in preparation size and irrigation [8]. On the other hand, Peters and Wesselink [9] demonstrated that more than $30 \%$ of the root canal walls remained untouched even by modern rotary $\mathrm{NiTi}$ instrumentation techniques. But not all the $\mathrm{Ni}-\mathrm{Ti}$ instruments can closely follow the original root canal path and procedural errors such as ledges, over-instrumentation and apical transportation could be present.

Mechanical debridement combined with antibacterial irrigation using $0.5 \%$ sodium hypochlorite can render $40-60 \%$ of the treated teeth bacteria negative [10-12]. In addition to mechanical debridement and antibacterial irrigation, dressing the canal with calcium hydroxide has been shown to increase the percentage of bacteria negative teeth to around $70 \%[13,14]$.

The aim of this study was to compare the instrumentation time efficiency of rotary and hand instrumentation performed on vital and necrotic human primary teeth.

\section{Materials and Methods}

This study took place at the Universidad Autónoma de Baja California, School of Dentistry in Tijuana, Mexico. The subjects review committee approved the study and all the participants were treated in accordance with the Helsinki Declaration (www.cirp.org/library/ethics/ helsinki). The study was developed between July 2011 and June 2013.

The main inclusion criteria were radiographic evidence of any sign of apical periodontitis or root resorption, teeth were the pulpotomy was started and teeth with diagnosis of pulpal necrosis confirmed by negative response to hot and cold tests. Thermal pulp testing was performed by the author, and radiographic interpretation was verified by two certified pedodontists.

The results from a pilot procedure were used to calculate the sample size. Calculation was performed using the two-sample paired t-test in the Bio Stat 4.0 software (two related samples, mean difference in log 10 colony-forming units before and after instrumentation, alpha 0.01). Fifteen teeth per group were required to obtain statistical power.

Patient selection was based on the following criteria: 1) the aims and requirements of the study were freely accepted by the parents; 2) primary molars with at least $11.0 \pm 1.0 \mathrm{~mm}$ of working length 3 ) Treatment was limited to patients in good health; 4) All teeth had vital and non-vital pulps without a sinus tract; 5) Excess of bleeding during a pulpotomy; 6) Presence of enough coronal tooth and root structure; 7) No prior pulpectomy treatment on the involved tooth, 8) absence of perforation in the internal and/or external furcation area and 9) No analgesics or antibiotics were used before the clinical procedures began.

Exclusion criteria were patients without inclusion requirements or failure to obtain parent's authorization, and were excluded if they were older than 7 years old, had a positive history of antibiotic use within the past month, diabetic, hemophilic or if the tooth had root resorption or been previously accessed and initiated a pulpectomy.

Patients aged 4-7 years of age were enrolled in this study, forty five teeth (19 maxillary and 26 mandibular teeth), which had a total of 102 canals and completely formed apices and of minimum $10 \mathrm{~mm}$ root length were selected. All selected teeth had mature apexes with no radiographic sign of root resorption (Table 1).

Of the 45 treated primary molars, 31 teeth were diagnosed as having chronic pulpitis, and 14 as having pulp necrosis that responded negative to hot and cold tests; and, clinically, all pulps were confirmed to be necrotic (no blood) on entrance into the pulp chamber. Informed consent was obtained and written by the parents from each patient in accordance with the approval of the study by the ethical board of the Universidad Autónoma de Baja California, Facultad de Odontología Tijuana, México. All clinical procedures and measurements were conducted by the author.

A \#0 periapical radiograph was taken for each tooth in buccolingual projection to allow proper selection. The selected teeth included 7 second maxillary molars (three canals each), 8 first maxillary molars (2 canals each), 4 central incisors (one canal each), 17 first mandibular molars ( 2 canals each), 9 second mandibular molars (three canals each) for a total of 45 teeth with 102 canals (Table 1).

A standard session time recommended by the pedodontists was approximately 20-35 minutes in length to allow for acceptable time for completion of treatment. All treatment was performed by the author. As a novel treatment in Pedodontics, were included electronic apex locator (EAL), EndoVac system and LightSpeed LSX instruments.

The teeth were randomly divided into 3 groups (each tooth was considered as an experimental unit): Group I $(n=15)$ : the root canals were prepared manually with K-files (Dentsply-Maillefer, Ballaigues, Switzerland) and "step back technique" up to size \#35.

Group II $(n=15)$ : the root canals were instrumented with rotary Light Speed LSX instruments (Discus Dental, Culver City, CA, USA). They were used to complete the canal preparation to a size \#50 for the anteriors and molar teeth to size \#40.

Group III $(n=15)$ : root canals were instrumented with Pro Taper file (Maillefer, Ballaigues, Switzerland) using SX, S1 to F2. 0.5\% NaOCl was used for irrigation. The instrumentation time was measured for all the procedures and the results were analyzed with student's t-test. All statistical procedures were computed with SPSS 21.0 (SPSS Inc.,

\begin{tabular}{|l|c|c|}
\hline \multicolumn{2}{|c|}{$\begin{array}{c}\text { No. of canals } \\
\text { Maxillary } \\
\text { Teeth - canals }\end{array}$} & $\begin{array}{c}\text { Mandibular } \\
\text { Teeth - canals }\end{array}$ \\
\hline Central Incisor & $4-4$ & $0-0$ \\
First Molar & $8-16$ & $17-34$ \\
Second Molar & $7-21$ & $9-27$ \\
Total & & \\
$19-41$ & & \\
$26-61$ & & \\
\hline
\end{tabular}

Table 1: Distribution of 45 teeth (102 canals). 
Chicago, IL, USA). The Student $t$ test was used to compare data whether there were statistically significant differences between the results obtained clinically. Significance was set at $\mathrm{p}<0.05$.

After local anesthesia by $2 \%$ lidocaine with 1:100,000 epinephrine (58 Rue du Pont de Creteil, Saint - Maur des Fossés F-94100, France) and rubber dam isolation the tooth was disinfected with $2.5 \% \mathrm{NaOCl}$ (Ultra bleach, Bentonville, AR, USA).

All caries were removed and endodontic access cavities made with sterile high speed carbide \# 331 (SS White. Lakewood, NJ). The cervical third of each canal was flared with a SX ProTaper file (Maillefer, Ballaigues, Switzerland).

Each canal was irrigated consequently with $2.0 \mathrm{cc} 0.5 \%$ sodium hypochlorite. The final rinse was aspirated but no attempt was made to dry the canals. Working length was established with the Root ZX Electronic Apex Locator (J Morita, Irvine, CA) and confirmed radiographically. The canals were negotiated and enlarged with K-files hand instruments (Dentsply-Maillefer, Ballaigues, Switzerland) until reaching an ISO \#20 at the working length.

The Root ZX was used in accordance with the manufacturer's instructions. The buccal clip was attached to the patient's lip, and the probe was connected to a stainless steel $15 \mathrm{~K}$-file. The file was advanced within the root canal to a point just beyond the major foramen, as indicated by the flashing APEX bar on the liquid crystal display of the EAL.

When the file was in position the LCD display showed a flashing bar between APEX and 1. Measurements were considered to be correct if the instrument remained constant for at least 4-5 seconds. A digital photograph was taken and stored in Adobe Photoshop 5.5 (Adobe Systems Inc., San Jose, CA, USA). The position of the file tip for each root canal were evaluated by two examiners, if the two examiners disagreed a third previously calibrated researcher was asked to make the final decision. The final WL was established to be $1 \mathrm{~mm}$ coronal to the major foramen [12].

For the group I the root canals were prepared manually with K-files and "step back technique" up to size \#35. For the Group II, Light Speed
LSX rotary instruments (Discus Dental, Culver City, CA, USA) were used to complete the canal preparation to a size \#50 for the anteriors and molars to size \#40.

For the group III the root canals were instrumented with ProTaper files using SX, S1 $(21 \mathrm{~mm})$ to $\mathrm{F} 2(21 \mathrm{~mm}) .0 .5 \% \mathrm{NaOCl}$ was used for irrigation; The original protocol suggested by ProTaper for permanent teeth was simplified for this study.

After completion of canal instrumentation, all canals were irrigated with distilled water for 30 seconds using the EndoVac irrigation system (Discus Dental, Culver City, CA, USA).

The EndoVac system is able to apply the irrigant to working length and evacuate it using apical negative pressure. The negative pressure avoids forcing the irrigant beyond the apex into the periapical tissues [15].

The canals were dried with sterile paper points and obturated at the same appointment using calcium hydroxide and iodoform paste [13] (Pearson Dental, Sylmar CA) by using pluggers or syringes (Messing Root Canal Gun, PD, Vevey, Switzerland). Access cavities of anterior teeth were etched and restored with Fuji IX (GC Corp, Tokyo, Japan). For posterior teeth, a buildup restoration was placed by using the same etching technique and Fuji IX or temporary metallic crown [3].

A stopwatch with alarm (http://stopwatch.onlineclock.net/) was used to record instrumentation time for each group (Table 2).

Children were recalled for clinical and radiographic examinations were evaluated, based on the criteria of Coll and Sadrian [13] at 6-month intervals for a follow-up period of 2 years. Teeth that exhibited no symptoms of pain, tenderness to percussion, swelling, sinus tract, or pathological mobility were judged clinically successful (42 teeth).

Teeth that showed no evidence of periradicular or interradicular radiolucency, internal or external root resorption, or periodontal ligament space widening were judged raiographically successful. Radiographic evidence of pulp canal obliteration was noted, but it was not regarded as failure.

\begin{tabular}{|l|c|c|c|c|}
\hline \multirow{2}{*}{$\begin{array}{l}\text { Type of } \\
\text { Instrumentation }\end{array}$} & $\begin{array}{c}\text { Anterior } \\
\text { teeth }\end{array}$ & $1^{\text {1st }}$ molars & $2^{\text {Maxillary }}$ & \multicolumn{2}{|c|}{ Mandibular } \\
\cline { 2 - 5 } molars & $20.14 \pm 5.4$ & $20.24 \pm 5.12$ \\
\hline Step Back & $7.22 \pm 1.91$ & $20.10 \pm 5.2$ & $10.45 \pm 4.77$ & $22.38 \pm 6.70$ \\
\hline LSX & $3.40 \pm 0.56$ & $8.03 \pm 3.80$ & $11.35 \pm 4.97$ & $10.40 \pm 3.62$ \\
\hline ProTaper & $3.49 \pm 0.59$ & $9.23 \pm 4.27$ & $9.77 \pm 3.99$ \\
\hline
\end{tabular}

$p=.005$

Table 2: Instrumentation time (minutes) for different groups of teeth

\begin{tabular}{|c|c|c|c|c|c|c|}
\hline \multirow{2}{*}{$\begin{array}{l}\text { Type of } \\
\text { Variable }\end{array}$} & \multirow{2}{*}{$\begin{array}{c}\text { Anterior } \\
\text { teeth }\end{array}$} & \multicolumn{2}{|c|}{ Maxillary } & \multicolumn{2}{|c|}{ Mandibular } & \multirow{2}{*}{$\Sigma$} \\
\hline & & $1^{\text {st }}$ molars & $2^{\text {nd }}$ molars & $1^{\text {st }}$ molars & $2^{\text {nd }}$ molars & \\
\hline $\begin{array}{l}\text { Preoperative } \\
\text { Root } \\
\text { Resorption }\end{array}$ & none & none & none & none & none & \\
\hline \multicolumn{7}{|l|}{ Pulpectomy lost } \\
\hline \multicolumn{7}{|l|}{ Length of fill: } \\
\hline Short & - & 2 & 1 & 2 & - & 5 \\
\hline Complete & 3 & 7 & 7 & 6 & 6 & 29 \\
\hline Long & 1 & 3 & 4 & 1 & 2 & 11 \\
\hline Pulpectomy exfoliated & none & none & none & none & none & \\
\hline Pulpectomy extracted & none & none & none & none & none & \\
\hline
\end{tabular}

$p=.005$

Table 3: Factors affecting pulpectomy success. 


\section{Results}

Of the 45 treated primary molars, 31 teeth were diagnosed as having chronic pulpitis (68.88\%), and 14 as having pulp necrosis (31.11\%). 6 to 12 -month recall examinations (Table 3 ). The clinical and radiographic success rates were $95 \%$ at the $12-24$ month follow-up time, and no obvious differences among different quadrants and tooth types were found. Only $4 \%(2 / 45)$ of the treated teeth experienced pain following the initial instrumentation, while $24 \%(11 / 45)$ had pain after the root canal filling.

However, the pain was mild and temporary and subsided in 1-2 days. A small area of expanded radiolucency was found in the furcal area in one case at the 6-month and in another case at the 12-month recalls, which were recorded as radiographic failure, although clinically no signs or symptoms were found (Table 3 ). The entire first visit, including local anesthesia, rubber dam placement, root canal preparations, was generally completed within 18-20 minutes. Of this time, canal preparation using rotary instruments only took approximately 8-12 minutes. Ledges or over instrumentation were not encountered, and neither instrument separation nor lateral perforation occurred.

With regard to canal filling quality, 29 cases $(64.44 \%)$ were flushfilled, 5 cases $(11.11 \%)$ were under-filled, and 11 cases $(24.44 \%)$ were over-filled. The over-filled Vitapex ${ }^{\bullet}$ was gradually resorbed within 9 months with no clinical symptoms or signs. There were no cases in which temporary restoration was found to be defective prior to crown placement (Table 3).

The mean time spent for rotary root canal preparation and hand preparation for the three groups was GI: $20.10 \pm 7.86$, GII: $9.37 \pm 2.19$ minutes and GIII: $10.45 \pm 4.77$ minutes, respectively. The difference between the three times was significant $(\mathrm{p}<0.001)$. The preparation time with group (I) K- files was significantly higher than in group (II and III) rotary instrumentation, with a $\mathrm{p}=0.005$ (Table 2).

\section{Discussion}

One of the most important concerns in pediatric dentistry is loss of primary molars leading to space loss. Several factors contribute to the clinical success of pulpectomy, such as biomechanical cleaning [16], type of restoration [6], number of visits [17] and root canal filling material.

The success of pulpectomy was related significantly to the amount of preoperative root resorption. Primary teeth with minimal or no preoperative root resorption had significantly higher pulpectomy success than those with excessive $(>1 \mathrm{~mm})$ resorption. This finding confirmed what the other PE studies had indicated $[13,14]$.

Excessive root resorption likely made it difficult to resolve the periapical infection with the PE procedure. The amount of preoperative root resorption seems to be the most important radiographic diagnostic criterion in determining whether a pulpectomy will likely succeed [13].

According to Finn [18] the main differences between deciduous and permanent teeth are that deciduous teeth are smaller in all dimensions than permanent teeth, and the mesiodistal/buccolingual crown ratio in temporary teeth is larger than in permanent teeth. Primary tooth dentin is softer and less dense than that of the permanent tooth, and the roots are shorter, thinner, and more curved, often with undetectable root tip resorption [1].

Chemo-mechanical preparation of the root canal includes both mechanical instrumentation and canal irrigation, and is principally directed toward the elimination of microorganisms from the root canal system [6]. Canal preparation is one of the most important phases of primary root canal treatment and is mainly aimed at the debridement of the canals [13].

Root canal instrumentation is essential to reduce infected content and create a root canal shape allowing for a well condensed root filling, the mean time spent for each step of the pulpectomy is essential to allows faster procedures with maintenance of quality and security, as well as reducing the patient's and professional's fatigue $[19,20]$.

The mean time spent for the instrumentation of groups II and III were similar and was lower than the one reported by Mortazavi [20]; their study was carried out in vivo study and was therefore more time consuming. Although Mortazavi [20] assessed clinical success rates, they also found no significant differences between rotary and hand instrumentation.

Because many pulpal ramifications cannot be reached mechanically, copious irrigation during cleansing and shaping must be maintained. The authors support the view that both chemical and mechanical cleaning affects root canal cleanliness. Furthermore, a key factor in the architecture of the rotary files may be their flute design [21].

Anatomic characteristics of root canals in deciduous teeth may be dramatically changed by the presence of physiologic or pathologic root resorption $[17,22,23]$, leading to problems related to root perforations.

The manual and rotary instruments produced less dentin removal on anterior teeth compared with other groups of teeth. This is explained by the root canal width and insufficient size of instruments [24].

Therefore, although our protocol recommends instrumentation up to size 40 , use of the combined techniques is suggested to allow more effective instrumentation. However, the produced dentin removal must be evaluated to establish the file size that may provide higher security to dental tissues and avoid excessive dentin removal and resulting increased fragility of the tooth structure.

Even though the fourth canal is not commonly present in maxillary molars [25], care must be taken considering the pattern of physiologic resorption guided by the position of the permanent tooth.

The lack of studies regarding root canal instrumentation in primary teeth and the results obtained in the present study underscore the importance of more research to develop safer and faster protocols for the treatment of deciduous teeth with compromised pulp tissue. Because endodontic treatment is the last option for maintenance of primary teeth affected either by caries or trauma in the oral cavity, it should follow the same biologic principles applicable to permanent teeth, aiming at success and thereby contributing to healthy development of the permanent dentition and the entire stomatognathic system. Instrumentation by manual or rotary techniques is safe to the deciduous tooth and permanent tooth bud, provided that all steps and cares are strictly followed, allowing disinfection of dental tissues and contributing to the repair of infectious and inflammatory processes.

\section{Conclusion}

Clinically, time efficacy in primary molar endodontics, especially with the unpredictability and difficulty of canal morphology, is invaluable. The use of rotary files in primary teeth has several advantages when compared with manual $K$ files: a) The efficiency in both, preparation time and root canal shape. b) A decreased working time, that helps maintain patient cooperation by diminishing the potential for tiredness. c) The shape of the root canal is more conical, favoring a higher quality of the root canal filling, and increasing clinical success. 
Citation: Vieyra JP, Enriquez FJJ (2014) Instrumentation Time Efficiency of Rotary and Hand Instrumentation Performed on Vital and Necrotic Human Primary Teeth: A Randomized Clinical Trial. Dentistry 4: 214. doi:10.4172/2161-1122.1000214

\section{Acknowledgement}

We thank Prof. Dr. Michael Hülsmann (Göttingen) and Dr. Clovis Monteiro Bramante for their valuable assistance in reviewing this manuscript.

\section{References}

1. Fuks AB (2002) Current concepts in vital primary pulp therapy. Eur J Paediat Dent 3: 115-120.

2. Tziafas D (2004) The future role of a molecular approach to pulp-dentinal regeneration. Caries Res 38: 314-320.

3. Fuks AB (2005) Pulp therapy for the primary dentition. In: Pediatric Dentistry: infancy through adolescence. St Louis, MO: Elsevier.

4. American Academy of Pediatric Dentistry (1996) Guidelines on pulp therapy for primary and immature permanent teeth. Reference manual 34: 12-13.

5. Rodd HD, Waterhouse PJ, Fuks AB, Fayle SA, Moffat MA (2006) Pulp therapy for primary molars. Int J Ped Dent 16: 15-23.

6. Moskovitz M, Sammara E, Holan G (2005) Success rate of root canal treatment in primary molars. J Dent 33: 41-47.

7. Silva LAB, Leonardo MR, Nelson-Filho P, Tanomaru JM (2004) Comparison of rotary and manual instrumentation techniques on cleaning capacity and instrumentation time in deciduous molars. J Dent Child 71: 45-47.

8. Kuo Cl, Wang YL, Chang HH, Huang GF, Lin CP, et al. (2006) Application of $\mathrm{Ni}$ Ti rotary files for pulpectomy in primary molars. J Dent Sci 1: 10-15

9. Peters LB, Wesselink PR (2002) Periapical healing of endodontically treated teeth in one and two visits obturated in the presence or absence of detectable microorganisms. Int Endod J 35: 660-667.

10. Kakehashi S, Stanley HR, Fitzgerald RJ (1965) The effects of surgical exposures of dental pulps in germfree and conventional laboratory rats. Oral Surg Oral Med Oral Pathol 20: 340-349.

11. Hülsmann M, Hahn W (2000) Complications during root canal irrigationliterature review and case reports. Int Endod J 33: 186-193.

12. Fuks $A B$, Papagiannoulis $L$ (2006) Pulpotomy in primary teeth: review of the literature according to standardized assessment criteria. Eur Arch Paediatr Dent 7: 64-71.
13. Coll JA, Sadrian R (1996) Predicting pulpectomy success and its relationship to exfoliation and succedaneous dentition. Pediat Dent 18: 57- 63.

14. Eidelman E, Holan G, Fuks AB (2001) Mineral trioxide aggregate vs. formocresol in pulpotomized primary molars: a preliminary report. Ped Dent 23:15-18.

15. Nielsen BA, Baumgartner CJ (2007) Comparison of the EndoVac system to needle irrigation of root canals. J Endod 33: 611-615.

16. Crespo S, Cortes O, Garcia C, Perez L (2008) Comparison between rotary and manual instrumentation in primary teeth. J Clin Ped Dent 32: 295-298.

17. McDonald RE, Avery DR (2000) Dentistry for the child and adolescent. (7thedn) St. Louis: Mosby.

18. Finn SB (1967) Morphology of the primary teeth. (3rdedn), WB Saunders Co, Philadelphia.

19. Pinheiro SL, Araujo G, Bincelli I, Cunha R, Bueno C (2012) Evaluation of cleaning capacity and instrumentation time of manual, hybrid and rotary instrumentation techniques in primary molars. Int Endod J 45: 379-385.

20. Mortazavi M, Abbasi A, Khodadadi E (2006) Comparison of canal cleansing time and pulpectomy success rate in deciduous molars by use of hand and rotary files. Dent J Shiraz Univ Med Sci 2: 111-119.

21. Khadivi Nia Javan N, Mohajeri Baradaran L, Azimi S (2007) SEM study of root canal walls cleanliness after Ni-Ti rotary and hand instrumentation. Iran Endod J 2: 5-10.

22. Prove SA, Symons AL, Meyers IA (1992) Physiological root resorption of primary molars. J Clin Pediatr Dent 16: 202-206.

23. Daito M, Kawahara S, Kato M, Okamoto K, Imai G, et al. (1991) Radiographic observations on root resorption in the primary dentition. J Osaka Dent Univ 25: 1-23.

24. Barr ES, Kleier DJ, Barr NV (2000) Use of nickel titanium rotary files for roo canal preparation in primary teeth. Pediatr Dent 22: 77-78.

25. Joseph T, Varma B, Mungara J (2005) A study of root canal morphology of human primary molars using computerised tomography: an in vitro study. $\mathrm{J}$ Indian Soc Pedod Prev Dent 23: 7-12. 